\title{
DISCUSSION ON D.C.M. DICKSON \& H.R. WATERS \\ MULTI-PERIOD AGGREGATE LOSS DISTRIBUTIONS FOR A LIFE PORTFOLIO
}

BY

BJøRn SUNDT

University of Bergen

In the present discussion we point out the relation of some results in Dickson \& Waters (1999) to similar results in Sundt (1998a, b).

We shall need some notation. For a positive integer $m$ let $\mathbb{N}_{m}$ be the set of all $m \times 1$ vectors with positive integer-valued elements and $\mathbb{N}_{m+}=\mathbb{N}_{m} \sim\{0\}$. A vector will be denoted by a bold-face letter and each of its elements by the corresponding italic with a subscript denoting the number of the elements; the subscript - denotes the sum of the elements. Let $\mathcal{P}_{m 0}$ be the class of probability functions on $\mathbf{N}_{m}$ with a positive probability at $\mathbf{0}$ and $\mathcal{P}_{m+}$ the class of probability functions on $\mathbb{N}_{m+}$. For $j=1, \ldots, m$ we introduce the $m \times 1$ vector $\mathbf{e}_{j}$ where the $j$ th element is one and all the other elements zero. We make the convention that summation over an empty range is equal to zero.

Let $g \in \mathcal{P}_{m 0}$ be the compound probability function with counting distribution with probability function $v \in \mathcal{P}_{10}$ and severity distribution with probability function $h \in \mathcal{P}_{m+}$; we shall denote this compound probability function by $v \vee h$. Sundt (1998a) showed that

$$
\begin{gathered}
g(\mathbf{x})=\frac{1}{x_{j}} \sum_{\mathbf{0}<\mathbf{y} \leq \mathbf{x}} g(\mathbf{x}-\mathbf{y}) y_{j} \sum_{i=1}^{y} \frac{\varphi_{v}(i)}{i} h^{i *}(\mathbf{y}) \quad\left(\mathbf{x} \geq \mathbf{e}_{j} ; j=1, \ldots, m\right) \\
g(\mathbf{x})=\frac{1}{x} \sum_{0<\mathbf{y} \leq \mathbf{x}} g(\mathbf{x}-\mathbf{y}) y \cdot \sum_{i=1}^{y \cdot} \frac{\varphi_{v}(i)}{i} h^{i *}(\mathbf{y}), \quad\left(\mathbf{x} \in \mathbb{N}_{m+}\right)
\end{gathered}
$$

where $\varphi_{v}$, denotes the De Pril transform of $v$, given by

$$
\varphi_{v}(t)=\frac{1}{v(0)}\left(t v(t)-\sum_{i=1}^{t-1} \varphi_{v}(i) v(t-i)\right) . \quad\left(t \in \mathbb{N}_{1}\right)
$$


Motivated by (2) Sundt (1998a) defined the De Pril transform $\varphi_{g}$ of $g$ by

$$
\varphi_{g}(\mathbf{x})=x \cdot \sum_{i=1}^{x .} \frac{\varphi_{v}(i)}{i} h^{i *}(\mathbf{x}) . \quad\left(\mathbf{x} \in \mathbb{N}_{m}\right)
$$

This defines the De Pril transform for all probability functions in $\mathcal{P}_{m 0}$. Insertion of (2) in (3) gives

$$
g(\mathbf{x})=\frac{1}{x_{\cdot}} \sum_{0<\mathbf{y} \leq \mathbf{x}} \varphi_{g}(\mathbf{y}) g(\mathbf{x}-\mathbf{y}), \quad\left(\mathbf{x} \in \mathbb{N}_{m+}\right)
$$

and by solving for $\varphi_{g}(\mathbf{x})$ we obtain

$$
\varphi_{g}(\mathbf{x})=\frac{1}{g(\mathbf{0})}\left(x . g(\mathbf{x})-\sum_{\mathbf{0}<\mathbf{y}<\mathbf{x}} \varphi_{g}(\mathbf{y}) g(\mathbf{x}-\mathbf{y})\right) . \quad\left(\mathbf{x} \in \mathbb{N}_{m}\right)
$$

Sundt (1998a) studies the De Pril transform defined in this way and found in particular that it is additive for convolutions.

As pointed out in Sundt (1998a), instead of basing our definition of the De Pril transform on (2) we could have based it on (1). Let $\varphi_{g}^{(j)}$ be the De Pril transform of $g$ obtained by this approach. For all $\mathrm{x} \geq \mathbf{e}_{j}$ we have

$$
\begin{aligned}
\varphi_{g}^{(j)}(\mathbf{x}) & =x_{j} \sum_{i=1}^{x_{0}} \frac{\varphi_{v}(i)}{i} h^{i *}(\mathbf{x})=\frac{x_{j}}{x} \varphi_{g}(\mathbf{x}) \\
g(\mathbf{x}) & =\frac{1}{x_{j}} \sum_{\mathbf{0}<\mathbf{y} \leq \mathbf{x}} \varphi_{g}^{(j)}(\mathbf{y}) g(\mathbf{x}-\mathbf{y}) \\
\varphi_{g}^{(j)}(\mathbf{x}) & =\frac{1}{g(\mathbf{0})}\left(x_{j} g(\mathbf{x})-\sum_{0<\mathbf{y}<\mathbf{x}} \varphi_{g}^{(j)}(\mathbf{y}) g(\mathbf{x}-\mathbf{y})\right) .
\end{aligned}
$$

The results of Sundt (1998a) are easily modified to the present situation. Result 1 in Dickson \& Waters (1999) follows from these formula and the additivity property of the De Pril transform with

$$
\begin{gathered}
w^{(j)}(\mathbf{x})=\varphi_{g}^{(j)}\left(\mathbf{x}+\mathbf{e}_{j}\right) \\
w_{i}^{(j)}(\mathbf{x})=\varphi_{g_{i}^{i_{i}}}^{(j)}\left(\mathbf{x}+\mathbf{e}_{j}\right)=n_{i} \varphi_{g_{i}}^{(j)}\left(\mathbf{x}+\mathbf{e}_{j}\right) \quad(i=1, \ldots, n)
\end{gathered}
$$

for $\mathrm{x} \in \mathbb{N}_{m}$ with $g_{i}$ denoting the probability function of the aggregate claims of a policy in class $i$. 
In Sundt (1998a) we also defined the De Pril transform in the multivariate case for more general functions than probability functions. In particular, this allows us to apply the theory also for approximations to distributions when the approximations are not necessarily distributions themselves. In Sundt (1998b) we extended to the multivariate case error bounds that had earlier been developed for such approximations in the univariate case, assuming that also the approximation has a positive mass at zero.

Let

$$
\varepsilon(g, \tilde{g})=\sum_{\mathbf{x} \in \mathbb{N}_{m}}|g(\mathbf{x})-\tilde{g}(\mathbf{x})| ; \quad \delta(g, \tilde{g})=\left|\ln \frac{g(\mathbf{0})}{\tilde{g}(\mathbf{0})}\right|+\sum_{\mathbf{x} \in \mathbb{N}_{m+}} \frac{\left|\varphi_{g}(\mathbf{x})-\varphi_{\tilde{g}}(\mathbf{x})\right|}{x .}
$$

with $g$ denoting the distribution and $\tilde{g}$ the approximation. Then

$$
\varepsilon(g, \tilde{g}) \leq e^{\delta(g, \tilde{g})}-1 .
$$

The proof of this inequality in Sundt (1998b) is a multivariate extension of the proof of the univariate case in Dhaene \& De Pril (1994). Their proof simplifies and unifies the deduction of the error bounds deduced in De Pril (1989) for the approximations of De Pril, Kornya, and Hipp.

If $g=*_{i=1}^{n} g_{i}^{n_{i *}}$ and $\tilde{g}=*_{i=1}^{n} \tilde{g}_{i}^{n_{i} *}$ with $g_{i}=v_{i} \vee h_{i}$ and $\tilde{g}_{i}=\tilde{v}_{i} \vee h_{i}$ for $i=1, \ldots, n$, then

$$
\delta(g, \tilde{g}) \leq \sum_{i=1}^{n} n_{i} \delta\left(g_{i}, \tilde{g}_{i}\right) \leq \sum_{i=1}^{n} n_{i} \delta\left(v_{i}, \tilde{v}_{i}\right) .
$$

In the situation of Dickson \& Waters (1999) $v_{i}$ is the Bernoulli distribution given by

$$
v_{i}(0)=p_{i} ; \quad v_{i}(1)=q_{i},
$$

which gives

$$
\varphi_{v_{i}}(t)=-\left(-\frac{q_{i}}{p_{i}}\right)^{t} \cdot \quad\left(t \in \mathbb{N}_{1+}\right)
$$

In the approximations of De Pril, Hipp, and Kornya in the univariate case the approximation $\tilde{v}_{i}$ is chosen such that $\varphi_{\tilde{v}_{i}}(t)=0$ for all integers $t$ greater than some fixed positive integer $r$. As pointed out in Sundt (1998b), the extension to the multivariate case is straight forward, and error bounds based on upper bounds for $\delta\left(v_{i}, \tilde{v}_{i}\right)$ are still valid.

In particular, in the De Pril approximation we have $\tilde{v}_{i}(0)=v_{i}(0)$ and $\varphi_{v_{i}}(t)=\varphi_{v_{i}}(t)$ when $t \leq r$. Furthermore, when $q_{i}<p_{i}$, we have

$$
\delta\left(v_{i}, \tilde{v}_{i}\right) \leq \frac{1}{r+1}\left(\frac{q_{i}}{p_{i}}\right)^{r+1} \frac{p_{i}}{p_{i}-q_{i}}
$$


(cf. e.g. Dhaene \& Sundt (1998), formula (8.11)), from which we obtain the same error bound as in Result 2 of Dickson \& Waters (1999). Furthermore, when considering how they in their formula (9) truncate the summation over $k$, we see that by replacing $v_{i}$ by $\tilde{v}_{i}$ we obtain the same approximation.

We conclude that the exact recursive method of Dickson \& Waters (1999) is obtained by a slight modification of methodology deduced in Sundt (1998a), a modification already indicated in that paper, and their approximation and its error bound is a special case of results in Sundt (1998b).

\section{REFERENCES}

DE PrIL, N. (1989). The aggregate claims distribution in the individual model with arbitrary positive claims. ASTIN Bulletin 19, 9-24.

Dhaene, J. \& De Pril, N. (1994). On a class of approximative computation methods in the individual model. Insurance: Mathematics and Economics 14, 181-196.

DhAENE, J. \& SunDT, B. (1998). On approximating distributions by approximating their De Pril transforms. Scandinavian Actuarial Journal, 1-23.

DiCKSON, D.C.M. \& WATERS, H.R. (1999). Multi-period aggregate loss distributions for a life portfolio. ASTIN Bulletin 29, *ـ*.

SundT, B. (1998a). The multivariate De Pril transform. Research paper 59, Centre for Actuarial Studies, University of Melbourne.

SUNDT, B. (1998b). On error bounds for multivariate distributions. Research paper 60, Centre for Actuarial Studies, University of Melbourne.

BJøRN SUNDT

Vital Forsikring ASA

PO Box 250

$N-1326$ Lysaker

Norway 